\title{
Prevalência de quedas e de fatores associados em idosos segundo etnia
}

\author{
The prevalence of falls and associated factors \\ among the elderly according to ethnicity
}

\begin{abstract}
Alexandre da Silva ${ }^{1}$
Henrique Herculano Faleiros ${ }^{1}$

William Akira Lima Shimizu ${ }^{2}$

Luciene de Morais Nogueira ${ }^{1}$

Luciane Lopes Nhãn ${ }^{1}$

Bruna Maiara Freitas da Silva ${ }^{1}$

Patrícia Miyuji Otuyama ${ }^{1}$
\end{abstract}

\footnotetext{
${ }^{1}$ Departamento de

Fisioterapia, Universidade Cruzeiro do Sul. Rua

Taiuvinha 31, Bairro São

Miguel Paulista, Campus São Miguel Paulista. 08060-040 São Paulo SP alexandre.silva@ cruzeirodosul.edu.br

${ }^{2}$ Instituto de Psicologia,

Universidade de São Paulo.
}

Abstract The historical and cultural characteristics reflect the Brazilian population. Elderly blacks are disadvantaged in socio-economic and demographic, clinical, functional and psychosocial aspects, reducing their social autonomy and functional independence. The decline in functional status due to variables associated with age and ethnicity can contribute to disabling events, such as falls. Socio-demographic, clinical and functional aspects related to falls were analyzed; Mobility, functional status and cognition were measured, with a statistical significance of pd"0.05. The sample of 196 elderly people was 48.5\% white, 28\% brown, 23.5\% black, with an average of 69.9 years. There was reduced mobility classified as a medium risk for falls in 60\% $(p<0.013)$ among the elderly. With reference to groups analyzed, there were significant differences between variables for family income $(p<0.029)$, the occurrence of falls $(p<0.006)$, fear of falls $(p<0.023)$ and nearfalls $(p<0.000)$. Blacks fall more often $(p<0.03)$. Statistical significance was revealed between ethnicity and self-reported occurrence of falls, fear of falling and the occurrence of near-falls, functional limitation and medium risk falls due to reduced mobility, with increased frequency of falls for elderly blacks.

Key words The elderly, Ethnic groups, Accidental falls
Resumo A característica histórico-cultural brasileira reflete a população. Idosos negros apresentam desvantagens nos aspectos socioeconômicos e demográficos, clínico-funcionais e psicossociais, reduzindo a autonomia social e a independência funcional. O declínio na condição funcional devido a idade e a variáveis associadas à etnia pode contribuir em eventos incapacitantes, como quedas. Foram coletados dados sociodemográficos, clinicos-funcionais, aspectos relacionados às quedas; Avaliou-se mobilidade, condição funcional e cognição. Estatística assumindo significância de $p \leq$ 0,05. Amostra de 196 idosos sendo 48,5\% brancos, 28\% pardos, 23,5\% negros. Média de 69,9 anos. Mobilidade reduzida classificando médio risco a quedas para 60\% ( $p<0,013)$ dos idosos. Referente aos grupos, significância entre variáveis para renda familiar $(p<0,029)$, ocorrência $(p<0,006) e$ medo de quedas $(p<0,023)$ e quase-quedas $(p<$ $0,000)$. Negros caem com maior frequência ( $p<$ 0,03). Significância estatística entre etnia autodeclarada e ocorrência de quedas, medo de cair e ocorrência de quase-quedas, limitação funcional e risco médio a quedas por mobilidade reduzida, frequência maior de quedas aos idosos negros.

Palavras-chave Idoso, Grupos étnicos, Acidente por quedas 


\section{Introdução}

De acordo com o Instituto Brasileiro de Geografia e Estatística (IBGE), o país se encontra em processo de envelhecimento com aumento gradativo de pessoas acima de 60 anos em consequência da redução da mortalidade e do aumento da expectativa de vida ${ }^{1}$.

Em 2007, os resultados da Pesquisa Nacional por Amostra de Domicílios (PNAD) mostraram que há no Brasil uma população de 20 milhões de idosos, que corresponde a $10,5 \%$ da população. Dentre estes, houve predominância de pessoas brancas ( 8,8 milhões), seguidos por 4,4 milhões de pardos e 1 milhão de negros. De acordo com esse senso, a população de mulheres idosas com mais de 60 anos, apresentou maior expressividade e longevidade em relação aos homens, entretanto, podem passar por período maior de debilidade física antes da morte ${ }^{1-3}$.

Um fato pouco estudado, mas que surge com importância significativa à saúde coletiva é a discussão sobre a condição de saúde dos idosos negros e a relação com sua situação demográfica, social e cultural com esta variável.

Segundo o Índice de Desenvolvimento $\mathrm{Hu}$ mano (IDH) brasileiro de 1999, o Brasil assumiu a 79a posição no ranking mundial, que o colocou numa condição de país miscigenado e de maioria branca. Ao analisar esses dados e considerando apenas a população branca, notou-se um salto do Brasil para a $43^{a}$ posição, comparando-o a países desenvolvidos considerados com IDH elevado, entretanto ao realizar essa mesma análise com a população negra, o Brasil decaiu para a $108^{a}$ equiparando-o a países pobres do Oriente Médio e África com IDH considerado médio, o que simboliza uma fraqueza econômica e social frente a outras nações.

Dados do IBGE revelaram a situação da população negra brasileira, com destaque para a inadequação do local e tipo dos domicílios dos negros direcionando-os a residirem em áreas sem condições de habitação e distantes de centros urbanos com carência de serviços públicos essenciais como escolas, unidades de saúde e saneamento, além do tipo de ocupação, nível escolar e renda familiar inferior, pois, a população negra, em sua maioria, faz parte da parcela populacional com pouca qualificação profissional e, consequentemente, com renda inferior para suprir carências como alimentação, vestuário e moradia ${ }^{1,2,4-6}$.

Alguns estudos demonstram que, apesar de não apresentar diferenças significativas entre brancos e negros no que diz respeito à autoper- cepção de saúde, prevalência de doenças crônicas e limitações funcionais, a população negra apresentou menores índices de acesso a programas de saúde básica e ambulatorial, entretanto, maior incidência nos casos de hospitalizações, o que representou problemas mais graves de saúde, sendo pior entre os homens ${ }^{1,2,4-6}$.

As atividades de cultura e lazer, também ficaram limitadas, assim como o local da habitação, devido às condições impróprias de áreas para práticas de atividades recreativas e físicas e ações de políticas públicas para implantação de programas de incentivo a atividades físicas e acesso cultural aos mais pobres ${ }^{1,2,4-6}$.

Já em relação à expectativa de vida, foi constatado que há maior longevidade na população brasileira negra, resultado do processo sanidade/enfermidade e retrato das condições materiais e sociais (meio ambiente físico, social, político e cultural em interação com as condições biológicas de cada ser humano), entretanto não suficiente para reduzir a disparidade quando comparada à população branca ${ }^{1,2,4-6}$.

Na população negra, a redução da capacidade funcional e as influências de fatores demográficos, socioeconômicos, culturais e psicossociais contribuem de maneira direta para eventos incapacitantes, como quedas, que se destacam por apresentarem graves consequências $s^{7-14}$.

Com o aumento da expectativa de vida, evidenciou-se o crescimento da incidência de doenças crônico-degenerativas (hipertensão arterial sistêmica, diabetes mellitus, osteoporose), distúrbios neurológicos associados ao envelhecimento (alteração proprioceptivas, visuais, vestibulares e mentais) e músculo-esqueléticos (diminuição de força e resistência muscular), que repercutem diretamente na redução de autonomia e independência à realização das atividades de vida diária (AVD) com consequente incapacidade, resultado de uma deficiência para desempenhar uma atividade considerada normal, ou desvantagem, caracterizada pelo prejuízo no desempenho de ações de acordo com a idade, o sexo, os fatores sociais e os culturais, por redução da capacidade funcional ${ }^{9}$.

Capacidade funcional definida como ausência de dificuldades no desempenho de atividades da vida cotidiana, pela Classificação Internacional de Comprometimento, Incapacidades e Desvantagens (ICIDH) da World Hearth Organization (WHO), neste contexto, torna-se importante fator protetor para a população geriátrica negra.

A queda definida como evento inesperado de mudança de posição para um nível mais baixo 
por perda do equilíbrio corporal pode provocar consequências imprevisíveis desde fraturas ósseas a risco de morte bem como medo de cair, restrição de atividades, declínio do estado de saúde e institucionalizações por ser causador potencial de incapacidade funcional e morbidade ${ }^{15-18}$.

No Brasil, cerca de 29\% dos idosos sofreram quedas num período de um ano. Alguns fatores podem ser indicativos para essa incidência, dentre eles, os que mais se destacam são: sexo feminino, idade acima de 75 anos, quedas recorrentes, comprometimentos nas atividades de vida diária (AVD), inatividade, uso de medicamentos e alterações de equilíbrio e mobilidade ${ }^{11,19,20}$.

Baseado no exposto, a condição social dos idosos negros brasileiros apresenta, de maneira geral, desvantagens em relação à população bran$\mathrm{ca}$, sendo esses fatores contribuintes para a redução de autonomia social e declínio da condição de saúde e independência funcional ${ }^{21}$, por dificuldade de acesso a serviços sociais, de lazer e, mais importante, de saúde.

Sendo assim, o objetivo deste estudo consistiu em avaliar a ocorrência de quedas e os fatores associados como sua frequência, e a relação entre mobilidade e funcionalidade sobre elas, a ocorrência de quase-quedas e o medo de cair e a relação entre aspectos socioeconômicos e demográficos sobre a prevalência de quedas em idosos de diferentes etnias autodeclaradas.

\section{Metodologia}

Tratou-se de estudo transversal analítico com amostra de 205 idosos de etnias negra, branca, parda ou amarela, autodefinida pelo entrevistado, durante questionário que abordou aspectos sociodemográficos, socioeconômicos, assim como questões sobre quedas. Os idosos inclusos possuíam idade igual ou superior a 60 anos, cognição preservada e deambuladores. Foram exclusos idosos com doenças psiquiátricas graves, exclusivamente cadeirantes e acamados.

A coleta de dados iniciou-se após aprovação pelo Comitê de Ética da Universidade Cruzeiro do Sul.

O método utilizado para colher os dados da pesquisa foi por conveniência. Os idosos foram abordados em centros comunitários gratuitos voltados à terceira idade em bairros da zona leste do município de São Paulo: Vila Ema, Vila Matilde e São Miguel Paulista.

Explicava-se a proposta e os objetivos da pesquisa para conhecimento da temática, caso hou- vesse aceite, fornecia-se Termo de Consentimento Livre e Esclarecido e iniciava-se a aplicação dos questionários e dos testes avaliativos.

A avaliação geral consistiu em analisar condições socioeconômicas e demográficaos (referentes à idade, ao sexo, ao estado civil, à escolaridade, ao arranjo familiar, à moradia, à ocupação e à renda familiar por habitante) e clínico-funcionais (envolveram capacidade funcional, condição clínica geral e doenças associadas).

Informações sobre quedas foram obtidas através de questionário com respostas de múltipla escolha desenvolvido pelo pesquisador principal com embasamento na literatura a fim de trazer ao estudo maior grau de especificidade. Pode-se avaliar a ocorrência e a frequência de quedas, o local, os motivos e os meios de ocorrência, as consequências como medo de cair e o evento de quase quedas - evento inesperado de instabilidade postural seguido por recuperação da estabilidade, não evoluindo para queda.

Os aspectos cognitivos foram avaliados pelo "Mini Exame do Estado Mental" (MEEM), que fornece imediatamente um indicador aceitável de possibilidade demencial por questões de aspectos cognitivos com pontuação máxima de 30 pontos $^{22}$. Os pontos de corte para o MEEM segundo o nível de escolaridade em nosso país são: analfabetos, 20 pontos; idade de 1 a 4 anos, 25 pontos; 5 a 8 anos, 26,5 pontos; de 9 a 11 anos, 28 pontos; indivíduos com escolaridade superior a 11 anos, 29 pontos $^{23}$.

A capacidade funcional foi analisada através da "Olders American Resources Service" (OARS $)^{24}$, questionário modificado com 15 perguntas designadas a obter informações sobre realização de AVD dos idosos. Considerou-se para cada tarefa funcional questionada desse instrumento quatro categorias de resposta possíveis ("sem dificuldade", "com pouca dificuldade", "muita dificuldade" ou "não realiza"). A seguir foi construída variável qualitativa com quatro categorias de dificuldade funcional, segundo número de AVD comprometidas: "nenhuma AVD comprometida", "1 a 5 AVD comprometidas", "6 a 10 AVD comprometidas" e "11 ou mais AVD comprometidas"25.

O “Timed Up and Go Test” (TUGT) tem como objetivo avaliar mobilidade e equilíbrio, no qual quantifica em segundos mobilidade funcional através da tarefa levantar de uma cadeira padronizada com apoio para as costas, braços de aproximadamente $46 \mathrm{~cm}$ de altura, caminhar percurso linear de 3 metros, virar-se e voltar-se rumo à cadeira sentando novamente ${ }^{26}$. Os grupos foram 
classificados, após a realização do teste, em três subgrupos, de acordo com o tempo gasto para realizá-lo: menos de 10 segundos, baixo risco de quedas; 10 a 20 segundos, médio risco de quedas e acima de 20 segundos, alto risco de quedas ${ }^{27}$.

Após obtenção e tabulação dos dados, realizou-se análise estatística descritiva simples para verificar a distribuição das informações de acordo com variáveis estudadas. Posteriormente, foram realizadas análises analíticas para comparar médias-teste t e ANOVA, e os testes de Fisher e qui-quadrado para as variáveis qualitativas. Assumiu para essa pesquisa um nível de significância de 5\%. Para realizar as análises estatísticas foi utilizado o programa Statistical Package for Windows (SPSS) versão 15.0.

\section{Resultados}

Para obtenção destes dados, fez-se o uso de um questionário sobre quedas desenvolvido pelos pesquisadores envolvidos no estudo, conforme mencionado em procedimento metodológico. O questionário mostrou boa especificidade à coleta dos dados em relação às experiências dos idosos frente às possíveis ocorrências de quedas e boa aplicabilidade por oferecer dados de simples coleta, ambas de aspectos importantes para a pesquisa.

A amostra foi composta por 205 idosos sendo autodeclarados como negros, brancos, pardos ou amarelos.

Indivíduos autodeclarados como amarelos foram avaliados, entretanto devido ao tamanho amostral pequeno que poderia ocasionar desvios, os indivíduos desta amostra foram excluídos da análise estatística totalizando uma amostra de 196 idosos.

Tratou-se de amostra heterogênea com média de idade de 69,9 $\pm 6,906$ anos sendo que 139 $(70,9 \%)$ eram do sexo feminino e $57(29,1 \%)$ do sexo masculino, autodeclaração em brancos ocorreu em $95(48,5 \%)$ dos idosos avaliados, pardos em 55 (28\%) e negros em 46 (23,5\%), com média de escolaridade de 4,4 anos de estudo completos e com vida conjugal em 87 (44,4\%).

Quando questionados a respeito da renda familiar, que deveria envolver a somatória de todas as pessoas que residem na casa do entrevistado totalizou $\mathrm{R} \$ 1.247,89$, sendo para a etnia branca, a média foi de $\mathrm{R} \$ 1.405,00$, na etnia parda de $\mathrm{R} \$ 1.255,91$ e na etnia negra de $\mathrm{R} \$ 913,85$ (ANOVA $\mathrm{p}=0,029)$, com renda provinda de pensão ou aposentadoria $(\mathrm{n}=155 / 79 \%)$, salário mensal ( $n=26 / 13,3 \%)$, salário mensal com apo- sentadoria conjugada ( $n=4 / 2 \%)$ ou outra condição não presente nas alternativas $(\mathrm{n}=11 / 5,7 \%)$ devido a suporte familiar.

A forma de moradia de $165(84,2 \%)$ é própria, $13(6,6 \%)$ alugada, 11 (5,6\%) dos filhos, 5 $(2,6 \%)$ de familiares ou amigos e $2(1 \%)$ se encontravam em outra situação.

No aspecto clínico funcional, quando questionados acerca de sua percepção de saúde, 106 (54\%) a classificaram como regular, $64(32,7 \%)$ como boa, 15 (7,7\%) como ruim, 7 (3,6\%) como excelente e somente $4(2 \%)$ dos idosos a classificaram como péssima. Quando questionados acerca de sua percepção de visão, os resultados obtidos foram um pouco mais negativos, 95 $(48,5 \%)$ a classificaram como regular, $51(26 \%)$ como boa, $34(17,3 \%)$ como ruim, $11(5,6 \%)$ como péssima e apenas 5 (2,6\%) como excelente.

A Tabela 1 apresenta dados relacionados à ocorrência de quedas sofridas nos últimos 12 meses. As variáveis que merecem destaque por apresentarem significância estatística foram: ocorrência de quedas, ocorrência de quase quedas e medo de quedas. Nas variáveis supracitadas, houve maior prevalência da população idosa negra com respostas afirmativas às problemáticas abordadas.

A variável frequência de quedas apresentou significância estatística (ANOVA p $=0,03$ ) analisando quantidade relacionada à etnia dos grupos autodeclarados sendo média de 2,3 quedas para os idosos negros, 1,3 para os brancos e 1,53 para os pardos.

Correlacionando-se ocorrência de quedas e quase quedas com medo de sofrer quedas, obteve-se em ambas boa significância estatística, gerando probabilidade de haver relação direta entre ter passado por uma experiência de queda e/ou quase queda influenciando numa maior prevalência de medo nos idosos entrevistados (Tabela 2).

Quando questionados sobre o grau de dificuldade na execução de determinadas atividades funcionais, vê-se na Tabela 3 que, a amostra demonstrou $64 \%$ dos idosos referindo não apresentar dificuldade, percentagem inferior quando recortados para amostragem da população negra (correspondente a 54\%) em relação à população branca (correspondente a $73 \%$ ) e parda (correspondente a 58\%), estando com pequena margem de diferença quando comparados os grupos e o nível de maior dificuldade.

O dado de significância estatística $(\mathrm{p}=0,015)$ se refere à execução das atividades funcionais com pouca dificuldade $(n=121)$ referidos por $78 \%$ dos idosos (sendo $23 \%$ negros, $53 \%$ brancos e $22 \%$ pardos). 
Tabela 1. Descrição dos fatores relacionados a quedas e suas características de acordo com a etnia autodeclarada.

\begin{tabular}{|c|c|c|c|c|}
\hline & Negros & Brancos & Pardos & $\mathbf{P}$ \\
\hline Ocorrência de queda & & & & $0,006^{*}$ \\
\hline Sim & $32(69,6 \%)$ & $39(41,1 \%)$ & $27(49,1 \%)$ & \\
\hline Não & $14(30,4 \%)$ & $56(58,9 \%)$ & $28(50,9 \%)$ & \\
\hline Local da queda & & & & 0,199 \\
\hline Em casa & $22(68 \%)$ & $16(42 \%)$ & $13(48 \%)$ & \\
\hline Na rua & $7(21 \%)$ & $18(47 \%)$ & $12(44 \%)$ & \\
\hline Outros & $3(9 \%)$ & $4(10 \%)$ & $2(7 \%)$ & \\
\hline Atividade realizada no momento da queda & & & & 0,164 \\
\hline Domésticas & $18(56 \%)$ & $14(36 \%)$ & $9(33 \%)$ & \\
\hline Rotineiras & $6(18 \%)$ & $15(39 \%)$ & $13(48 \%)$ & \\
\hline Outros & $8(25 \%)$ & $9(23 \%)$ & $5(18 \%)$ & \\
\hline Motivo da queda & & & & 0,234 \\
\hline Escorregou & $16(50 \%)$ & $13(34 \%)$ & $9(33 \%)$ & \\
\hline Tropeçou & $5(15 \%)$ & $17(44 \%)$ & $11(40 \%)$ & \\
\hline Desequilibrou & $8(25 \%)$ & $6(15 \%)$ & $4(14 \%)$ & \\
\hline Outros & $3(9 \%)$ & $2(5 \%)$ & $3(11 \%)$ & \\
\hline Ocorrência de quase quedas & & & & $0,000^{*}$ \\
\hline Sim & $41(89,1 \%)$ & $52(54,7 \%)$ & $36(65,5 \%)$ & \\
\hline Não & $5(10,9 \%)$ & $43(45,3 \%)$ & $19(34,5 \%)$ & \\
\hline Apresentou medo de quedas & & & & $0,023^{*}$ \\
\hline Sim & $43(93 \%)$ & $71(75 \%)$ & $41(75 \%)$ & \\
\hline Não & $3(7 \%)$ & $24(25 \%)$ & $14(25 \%)$ & \\
\hline
\end{tabular}

(*) Valor de significância estatística para $\mathrm{p}<0,05$

Tabela 2. Verificação das variáveis ocorrência de queda e quase-queda associadas ao medo de cair.

\begin{tabular}{|c|c|c|c|c|c|c|}
\hline & \multicolumn{3}{|c|}{ Ocorrência de queda } & \multicolumn{3}{|c|}{ Ocorrência de quase queda } \\
\hline & Sim & Não & $\mathrm{p}$ & Sim & Não & $\mathbf{p}$ \\
\hline \multicolumn{7}{|c|}{ Medo de queda } \\
\hline Sim & $86(55,5 \%)$ & $69(44,5 \%)$ & $0,003^{\star}$ & $113(72,9 \%)$ & $42(27,1 \%)$ & $0,000^{*}$ \\
\hline Não & $12(29,3 \%)$ & $29(70,7 \%)$ & & $16(39 \%)$ & $25(61 \%)$ & \\
\hline
\end{tabular}

${ }^{*}$ Valor de significância estatística para $\mathrm{p}<0,05$

$\mathrm{Na}$ avaliação dos dados encontrados no TUGT, também obteve-se significância estatística demonstrando que grande parte da amostra $(\mathrm{n}=117 / 60 \%)$ se encontra com mobilidade moderadamente reduzida estando na classificação de médio risco a quedas, conforme Tabela 4.

A média de escolaridade encontrada na população estudada foi de 4,4 anos de estudo. Quando correlacionados anos de escolaridade com resultado obtido através do MEEM, obteve-se significância para grupo que apresentou média de 5 a 8 anos de estudo que deveria ter pontuação média de 26,5 devido grau de escolaridade (Tabela 5).

\section{Discussão}

As avaliações ocorreram em diversos bairros no intuito de abranger diferentes classes socioeconômicas e demográficas. Pressupõe-se que o local de abordagem seja fator de diversidade étnica, diferentes condições socioeconômicas e demográficas, impactando em desníveis sociais como escolaridade, moradia, acesso à saúde, lazer e renda per capita, adicionando, assim, a classe social como variável importante do estudo presente.

Os dados sobre etnia neste estudo podem ser comparados com os apresentados em estudos prévios os como de Berquó ${ }^{28}$ e de Guccione ${ }^{29} \mathrm{com}$ 
Tabela 3. Caracterização quantitativa de atividades de vida diária e referência de nível de execução segundo OARS.

\begin{tabular}{|c|c|c|c|c|c|}
\hline & \multicolumn{3}{|c|}{ Etnia } & \multirow[b]{2}{*}{ Total } & \multirow[b]{2}{*}{$\mathbf{P}$} \\
\hline & Negros & Brancos & Pardos & & \\
\hline Sem Dificuldade & & & & & 0,061 \\
\hline Até 5 atividades & $5(42 \%)$ & $2(16 \%)$ & $5(42 \%)$ & $12(100 \%)$ & \\
\hline 6 a 10 atividades & $14(26,5 \%)$ & $21(39,6 \%)$ & $18(33,9 \%)$ & $53(100 \%)$ & \\
\hline 11 a 15 atividades & $25(19,6 \%)$ & $70(55,2 \%)$ & $32(25,2 \%)$ & $127(100 \%)$ & \\
\hline Pouca Dificuldade & & & & & $0,015^{\star}$ \\
\hline Até 5 atividades & $29(23,9 \%)$ & $65(53,7 \%)$ & $27(22,4 \%)$ & $121(100 \%)$ & \\
\hline 6 a 10 atividades & $12(44,5 \%)$ & $5(18,5 \%)$ & $10(37 \%)$ & $27(100 \%)$ & \\
\hline 11 a 15 atividades & $2(28,5 \%)$ & $2(28,5 \%)$ & $3(43 \%)$ & $7(100 \%)$ & \\
\hline Muita Dificuldade & & & & & 0,421 \\
\hline Até 5 atividades & $9(16,3 \%)$ & $28(50,9 \%)$ & $18(32,8 \%)$ & $55(100 \%)$ & \\
\hline 6 a 10 atividades & $2(50 \%)$ & $1(25 \%)$ & $1(25 \%)$ & $4(100 \%)$ & \\
\hline 11 a 15 atividades & $0(0 \%)$ & $1(100 \%)$ & $0(0 \%)$ & $1(100 \%)$ & \\
\hline
\end{tabular}

Valor de significância estatística para $\mathrm{p}<0,05$.

Tabela 4. Relação qualitativa dos valores mensurados no teste TUGT com etnia autodeclarada relacionado ao risco de quedas.

\begin{tabular}{lrrrrr}
\hline & \multicolumn{4}{c}{ Etnia } & \\
\cline { 2 - 3 } \multicolumn{1}{c}{ TUGT } & \multicolumn{1}{c}{ Negro } & \multicolumn{1}{c}{ Branco } & \multicolumn{1}{c}{ Pardo } & Total & P \\
\hline Baixo risco (0 a 10 segundos) & $8(10,7 \%)$ & $42(56 \%)$ & $25(33,3 \%)$ & $75(100 \%)$ & $0,013^{*}$ \\
Médio risco (11 a 20 segundos) & $36(30,7 \%)$ & $51(43,6 \%)$ & $30(25,7 \%)$ & $117(100 \%)$ \\
Alto risco (21 a 30 segundos) & $2(50 \%)$ & $2(50 \%)$ & $0(0 \%)$ & $4(100 \%)$ \\
Total & $46(23,5 \%)$ & $95(48,5 \%)$ & $55(28 \%)$ & $196(100 \%)$ \\
\hline
\end{tabular}

Valor de significância estatística para $\mathrm{p}<0,05$.

Tabela 5. Categorização das pontuações esperadas do MEEM segundo anos de escolaridade e etnia referida

\begin{tabular}{|c|c|c|c|c|}
\hline & Negro (n) & Branco (n) & Pardo (n) & $\mathrm{p}$ \\
\hline Analfabeto & & & & 0,393 \\
\hline$<20$ pontos & 1 & 3 & 2 & \\
\hline$>20$ pontos & 0 & 2 & 4 & \\
\hline $1-4$ anos & & & & 0,615 \\
\hline$<25$ pontos & 16 & 30 & 19 & \\
\hline$>25$ pontos & 18 & 23 & 14 & \\
\hline $5-8$ anos & & & & $0,000^{*}$ \\
\hline$<26,5$ pontos & 6 & 4 & 8 & \\
\hline$>26,5$ pontos & 5 & 25 & 2 & \\
\hline Superior a 8 anos & & & & 0,426 \\
\hline$<28$ pontos & 0 & 5 & 2 & \\
\hline$>28$ pontos & 0 & 3 & 3 & \\
\hline
\end{tabular}

Valor de significância estatística para $\mathrm{p}<0,05$.

maior porcentagem de idosos brancos em relação a negros e pardos. Assim como Camarano ${ }^{30}$, referimos uma dificuldade da autodeclaração na composição da amostra por cor ou raça. A predominância da população branca permanece em evidência. $^{31}$ 
Semelhante também são os dados relacionados ao sexo. Neste estudo obteve-se uma amostra pequena de idosos comparados às idosas. Segundo Veras ${ }^{32}$, o processo de feminização da velhice pode justificar longevidade por diversos fatores como biológicos devido aos hormônios femininos exercerem fator protetor; comportamentais/hábitos de vida, menor exposição a fatores de riscos como fumo, álcool e acidentes automobilísticos e de trabalho; maior procura por acesso aos serviços de saúde; e influência social refletindo na composição de famílias chefiadas por mulheres, urbanização e contribuição na renda familiar.

O fato de viverem mais não significa estarem livres de doenças. Maior expectativa de vida faz com que passem pela experiência de debilitação biológica e doenças crônicas enquanto os homens acabam morrendo antes devido acidentes cardiovasculares, por exemplo ${ }^{3}$. Evidências apontam expressiva parcela de morbidade na população feminina, além de outros fatores associados, como viuvez e desvantagem socioeconômica, favorecendo maior participação e exposição de atividades extradomiciliares, que, por sua vez, podem influenciar neste processo como ciclo de desvantagem feminina ${ }^{30-34}$.

Quando discutido a respeito do perfil socioeconômico da população, sabe-se que o suporte social é capaz de exercer papel essencial capaz de promover e manter saúde física e mental. Entretanto, existem divergências em relação à condição socioeconômica dos diversos grupos, significando variações de classe na natureza e na quantidade em que o suporte social esta disponível. Sendo assim, é compreensivo concluir o fato de que indivíduos em classes sociais menos favorecidas seriam mais isolados e aparentariam receber menos assistência quando comparados a outros grupos que possuam classes sociais mais altas ${ }^{35}$.

Sobre a temática classes sociais, a discussão baseia-se na questão de receberem menos assistência, como foi supracitado, logo, pode-se questionar o fato de aparentarem estar em desvantagem em termos de saúde em decorrência dessa situação. Esses grupos estudados, que apresentam baixo status socioeconômico, têm saúde mais pobre. No caso, pessoas vivendo sob realidade da pobreza estão mais expostas a riscos físicos, químicos, bioquímicos, biológicos e até psicológicos que afetariam sua saúde. Estilo de vida mais saudável promoveria melhor qualidade de vida, auxiliaria na manutenção e são mais facilmente encontrados em classes mais altas, apesar de evidencias apontarem que a conquista de hábitos mais saudáveis tem começado a se difundir ${ }^{36}$. $\mathrm{O}$ encontrado na literatura demonstra ter correlação com os dados obtidos na pesquisa, pois, quando analisados os resultados, percebese que a população idosa negra, especificamente, apresentou menor renda familiar quando comparada aos outros grupos.

A partir dos resultados obtidos, a variável etnia autodeclarada assumiu pouca dificuldade na realização de AVD e médio risco à queda devido à mobilidade reduzida. Tais resultados podem ser representativos quando visualizados com as variáveis: ocorrência de quedas, ocorrência de quase-quedas e medo de cair.

Os grupos apresentaram ocorrência de quedas num período de 12 meses, sendo inferido que idosos negros apresentaram-se maior porcentagem em relação aos outros grupos étnicos autodeclarados, assim como para variáveis quasequedas e medo de cair. A importância dessa variável é dada pelo fato de alguns estudos demonstrarem que o envelhecimento acarreta redução da amplitude de movimentos ${ }^{37}$, sendo essa diminuição da flexibilidade associada à ocorrência de quedas, principalmente devido à diminuição de mobilidade no quadril, joelhos, tornozelos e coluna vertebral, gerando alterações na marcha e dificuldades no desempenho de funções ${ }^{38}$.

Em decorrência de todos esses transtornos acarretados ao idoso, a literatura indica que 30\% cai pelo menos uma vez em 12 meses, dos quais metade ocorre de forma recorrente ${ }^{39,40}$. Verificado neste estudo prevalência estatística de um episódio de queda nos últimos 12 meses $(\mathrm{p}=0,006)$ e também mostrado frequência de quase-quedas neste mesmo período, dados significantes quando relacionados à recorrência. Independente de etnia, idosos caíram neste período com significância para frequência de quedas recorrentes entre grupos, índice que pode ter reflexo sobre quase-quedas e medo de cair. Destaque ao grupo étnico negro cuja presença de maior porcentagem nas variáveis, ocorrência de quedas, frequência de quedas ( $\mathrm{p}=0,03)$, quase-quedas e medo de cair, foi encontrada. Em contraposição, no estudo de Faulkner et al. ${ }^{41}$, entre as idosas acima de 75 anos, houve maior prevalência de quedas para brancas quando comparado às negras. Hanlon et al. ${ }^{42}$, obteve resultado semelhante ao estudo supracitado, verificou que idosas brancas estariam mais propensas a apresentar pelo menos um episódio de queda em 12 meses antecedentes à pesquisa quando comparadas às negras.

Também é importante salientar que dados do Censo 2000 demonstrou que mais de $80 \%$ 
dos idosos possuem ao menos uma doença crônica e quase metade necessita de algum auxílio para realizar ao menos uma de suas AVD e quase $10 \%$ dos idosos tem algum tipo de dependência, favorecendo ocorrência de pelo menos um quadro de queda ${ }^{1,43}$.

Percebe-se que é elevado o número de idosos que caem, acarretando em mudanças em diversos aspectos do seu dia a dia, tanto pela queda, como pelo medo de recorrência. A seriedade apresentada pelos episódios de queda é percebida pelos impactos causados: restrição de atividades, isolamento social, declínio na saúde e aumento do risco de institucionalização ${ }^{44,45}$. Com isso, o medo de voltar a cair, ou "síndrome pósqueda" é visto como segunda maior consequência citada pelos idosos, encontrado no estudo e relatado por outros autores ${ }^{46-48}$.

O medo do idoso após a queda acarreta não somente o medo de recorrência, mas também de machucar-se, da hospitalização, da imobilização, do declínio de saúde e, principalmente, de tornar-se dependente para autocuidado ou realização de AVD, ou seja, medo das consequências inerentes à queda ${ }^{49}$. Segundo Owings et al. ${ }^{50}$ quando idosos caem e são hospitalizados, é comum ocorrer imobilismo e até mesmo invalidez. Geralmente, após queda, há tendência a limitar AVD e AIVD, repercutindo negativamente no bem-estar e na qualidade de vida, necessitando cada vez mais de maior suporte familiar. Entretanto, pôde ser demonstrado neste estudo a pouca limitação funcional na execução das AVD e na manutenção da participação social, representando ponto positivo à população estudada seja para brancos ou não.

Fato curioso que foi encontrado no presente estudo, e no de Berg et al. ${ }^{51}$, os idosos caem durante atividades simples como caminhar e limpar, quando o esperado é maior ocorrência de quedas durante realização de atividades com maior risco como subir e descer escadas. Apenas dois casos de queda ocorreram desta forma, um por subir em cadeira para alcançar objetos e outro por subir em andaime. Problemas ambientais também representaram causas frequentes, em que tropeços e escorregões foram grande causa de quedas ${ }^{46,51}$.

Podemos salientar, saber o local onde ocorreu a queda é importante para poder identificar fatores ambientais causadores como desníveis de solo, pisos escorregadios e obstáculos no percurso. Rocha e Cunha ${ }^{52}$ verificaram maior proporção de queda no lar das idosas. Quando Campbell et al. ${ }^{53}$ decidiram investigar ocorrência de acidentes com idosos que vivem na comunidade, comprovaram que 'queda' representou $51 \%$ dos acidentes e, mais uma vez, ocorreram prioritariamente na residência do idoso ( $44 \%$ das quedas). Salientase que estes dados são elucidativos ao planejamento de medidas ambientais e clínicas preventivas de quedas em idosos tanto pela alta incidência como pela gravidade variando desde pequenas escoriações até mesmo risco de morte ${ }^{46}$.

Alguns fatores que são mencionados como prejudiciais e potenciais geradores de quedas, com repercussão negativa à funcionalidade, podem representar fatores protetores aos idosos. Vivenciar evento de quase-quedas e a sensação de medo de cair seriam prejudiciais se acompanhados de exclusão à participação, à interação social e ao declínio funcional. Entretanto, segundo os dados obtidos e os relatos dos idosos, essas condições talvez possam servir para se protegerem das quedas, já que conhecendo as consequências e tendo uma experiência prévia desse problema, se tornariam mais cuidadosos.

\section{Conclusão}

Verificou-se significância entre etnia autodeclarada e ocorrência de quedas em período de 12 meses, medo de cair e quase-quedas prevalente entre os grupos, com pouca dificuldade na realização de AVD e risco médio a quedas por mobilidade reduzida, com frequência maior de quedas aos idosos negros entre os grupos estudados.

\section{Colaboradores}

A Silva participou na orientação e no auxílio sobre idealização, elaboração, coordenação, estatística e finalização; HH Faleiros participou na idealização, elaboração, coordenação, pesquisa de campo, estatística e finalização; WAL Shimizu na orientação e no auxílio sobre idealização, elaboração, coordenação, estatística e finalização; LM Nogueira, LL Nhãn, BMF Silva e PM Otuyama na pesquisa de campo e na análise dos dados. 


\section{Referências}

1. Instituto Brasileiro de Geografia e Estatística (IBGE). Censo 2000. Rio de Janeiro: IBGE; 2000.

2. Instituto Brasileiro de Geografia e Estatística (IBGE). Sintese de Indicadores Sociais - uma análise das condições de vida da população brasileira de 2007. Rio de Janeiro: IBGE; 2007.

3. Nogales AMV. A Mortalidade da População Idosa no Brasil. In: Como Vai? População Brasileira. Ano III no 3; 1998; Brasília. p. 24-32.

4. Sant'Anna W. Desigualdades étnico/raciais e de gênero no Brasil. Jornal da Rede, Rede Saúde [periódico na Internet]. $2001 \mathrm{Mar}^{\circ}{ }^{23}$ [acessado 2009 maio 28]. Disponível em: http://www.redesaude.org.br/ jornal/html/jr23-wania.html.

5. Berquó E. A Importância dos estudos sobre população negra. Jornal da Rede, Rede Saúde [periódico na Internet]. $2001 \mathrm{Mar}$ (23) [acessado 2009 maio 28]. Disponível em: http://www.redesaude.org.br/ jornal/html/jr23-elza.html.

6. Werneck J. Incorporação das dimensões de gênero e de igualdade racial e étnica nas ações de combate à pobreza e à desigualdade. In: Articulação de Organizações de Mulheres Negras Brasileiras (AMNB); 2006; Rio de Janeiro. p. 4-21.

7. Jette AM, Branch LG. Impairment and disability in the aged. J Chronic Dis 1985; 38(1):59-65.

8. Chamie M. The status and use of the International Classification of Impairments, disabilities and Handicaps (ICIDH). World Health Stat Q 1990; 43(4):273280.

9. Amiralian MLT, Pinto EB, Ghirardi MIG, Lichtig I, Masini EFS, Pasqualin L. Conceituando deficiência. Rev Saude Publica 2000; 34(1):97-103.

10. Oliveira F. Saúde da população negra: Brasil ano 2001. Brasília: Organização Pan-Americana da Saúde (OPAS); 2003.

11. Rosa TEC, Benício MHD, Latorre MDR, Ramos LR. Fatores determinantes da capacidade funcional entre idosos. Rev Saude Publica 2003; 37(1):40-48.

12. Maciel ACC, Guerra RO. Fatores Associados à Alteração da Mobilidade em Idosos Residentes na Comunidade. Rev. bras. fisioter. 2005; 9(1):17-23.

13. Grimley-Evans J. Prevention of age-associated loss of autonomy: epidemiological approaches. J Chronic Dis 1984; 37(5):353-363.

14. Gitlin LN, Hauck WW, Dennis M, Schulz R. Depressive symptoms in African American and White older adults with functional difficulty: the role of control strategies. J Am Geriatr Soc. 2007; 55(7):10231030.

15. Cunha UGV, Guimarães RM. Sinais e sintomas do aparelho locomotor. In: Guimarães RM, Cunha UG de V, organizadores. Sinais e sintomas em geriatria. Rio de Janeiro: Revinter; 1989. p. 141-154.

16. Rizzo JA, Friedkin R, Williams CS, Nabors J, Acampora D, Tinetti ME. Health care utilization and costs in a Medicare population by fall status. Med Care 1998; 36(8):1174-1188.

17. Close J, Elis M, Hooper R, Gluckmsn E, Jackson S, Cameron S. Prevention of falls in the elderly trial (PROFET): a randomised controlled trial. Lancet 1999; 353(9147):93-97.
18. Moura RN, Santos FC, Driemeier M, Santos LM, Ramos LR. Quedas em idosos: fatores de risco associados. Gerontologia 1999; 7(2):15-21.

19. Tinetti ME, Speechley M, Ginter SF. Risk factors for falls among elderly persons living in the community. N Engl J Med 1988; 319(26):170-177.

20. Perracini MR, Ramos LR. Fatores associados a quedas em uma coorte de idosos residents na comunidade. Rev Saude Publica 2002; 36(6):709-716.

21. Bastos JL, Peres MA, Peres KG, Dumith SC, Gigante DP. Diferenças socioeconômicas entre autoclassificação e heteroclassificação de cor/raça. Rev Saude Publica 2008; 42(2):324-334.

22. Folstein MF, Folstein SE, McHugh PR. Mini-Mental State. A practical Method for grading the cognitive state of patients for the clinician. J Psychiatr Res1975; 12(3):189-198.

23. Brucki SMD, Nitrini R, Caramelli P, Bertolucci PHF, Okamoto IH. Sugestões Para o Uso do Mini-Exame do Estado Mental no Brasil. Arq Neuropsiquiatr 2003; 61(3-B):777-781.

24. Blay SL, Ramos LR, Mari JJ. Validity of Brazilian version of Olders American Resources Service (OARS) mental health screening questionnaire. $J$ Am Geriatr Soc 1988; 36(8):687-692.

25. Siqueira AB, Cordeiro RC, Perracini MR, Ramos LR. Impacto funcional da internação hospitalar de pacientes idosos. Rev Saude Publica 2004; 38(5):687694.

26. Podsiadlo D, Richardson S. The Timed Up \& Go: a test of basic functional mobility for frail elderly persons. J Am Geriatr Soc 1991; 39(2):142-148.

27. Guimarães LHCT, Galdino DCA, Martins FLM, Vitorino DFM, Pereira KL. Comparação da propensão de quedas entre idosos que praticam atividade física e idosos sedentários. Revista Neurociências $2004 \mathrm{Abr} / J u n$ [acessado 2012 jul 12];12(2): [cerca de 2 p.]. Disponível em: http://www.unifesp.br/ dneuro/neurociencias/vol12_2/quedas.htm

28. Berquó ES. Ainda a questão da esterilização feminina no Brasil. In: Giffin K, organizador. Questões da saúde reprodutiva. Rio de Janeiro: Fiocruz; 1999. p. 113-126.

29. Guccione AA. Avaliação Funcional do Idoso. In: Guccione À, organizador. Fisioterapia Geriátrica. 2a edição. Rio de Janeiro: Guanabara Koogan; 2002. p. 114-124.

30. Camarano AA, El Ghaouri SK. Família com idosos: ninhos vazios? Rio de Janeiro: IPEA; 2003.

31. Camarano AA, El Ghaouri SK. Idosos brasileiros: que dependência é essa? In: Camarano AA, organizador. Muito além dos 60: os novos idosos brasileiros. Rio de Janeiro: IPEA; 1999.

32. Veras RP. País jovem com cabelos brancos: a saúde do idoso no Brasil. Rio de Janeiro: Relume Dumará/UERG; 1994.

33. Camarano AA. Mulher idosa: suporte familiar ou agente de mudança? In: Camarano AA. Estudos Avançados, no 49: USP; 2003. p. 35-63. 
34. Neri AL. Velhice e qualidade de vida na mulher. In: Neri AL, organizador. Desenvolvimento e envelhecimento: perspectivas biológicas, psicológicas e sociológicas. Campinas: Papirus; 2001. p. 161-200.

35. Krause N, Borawisk-Clarck E. Social class differences in social support among older adults. The Gerontologist 1995; 35(4):498-508.

36. Cockerham W, Sahrp K, Wilcox J. Aging and perceived health status. J Gerontol 1990; 38(3):349-355.

37. Adams K, O'Shea P, O'Shea KL. Aging: its effects on strength, power, flexibility, and bone density. Strength Cond J 1999; 21(2):65-77.

38. Guimarães JMN, Farinatti PTV. Análise descritiva de variáveis teoricamente associadas ao risco de quedas em mulheres idosas. Rev Bras Med Esporte 2005; 11(5):299-305.

39. Tinetti ME. Falls in Elderly Persons. $N$ Engl J Med 2003; 348(1):42-49.

40. Liu-Ambrose T, Khan KM, Eng JJ, Janssen PA, Lord SR, McKay HA. Resistance and agility training reduce fall risk in women aged 75 to 85 with low bone mass: a 6-month randomized, controlled trial. J Am Geriatr Soc 2004; 52(5):1-9.

41. Faulkner KA, Cauley JA, Zmuda JM, Landsittel DP, Nevitt MC, Newman AB, Studenski SA, Redfern MS. Ethnic Differences in the Frequency and Circumstances of Falling in Older Community-Dwelling Women. JAGS 2005; 53(10):1774-1779.

42. Hanlon JT, Landerman LR, Fillenbaum GG, Studenski S. Falls in African American and White Community-Dwelling Elderly Residents. J Gerontol A Biol Sci Med Sci 2002; 57(7):M473-M478.

43. Pavarini SCI, Neri AL. Compreendendo Dependência, Independência e Autonomia no Contexto Domiciliar: Conceitos, Atitudes e Comportamentos. In: Duarte YAO, Diogo MJD, organizadores. Atendimento Domiciliar em Enfoque Gerontológico. São Paulo: Editora Atheneu; 2000. p. 49-69.

44. Carter ND, Kannus P, Khan KM. Exercise in the prevention of falls in older people: a systematic literature review examining the rationale and the evidence. Sports Med 2001; 31(6):427-438.
45. Stalenhoef PA, Diederiks JP, Knottnerus JA, Witte LP, Crebolder HF. The construction of a patient record-based risk model for recurrent falls among elderly people living in the community. Family Practice 2000; 17(6):490-496.

46. Fabrício SCC, Rodrigues RAP, Junior MLC. Causas e conseqüências de quedas de idosos atendidos em hospital público. Rev Saude Publica 2004; 38(1):9399.

47. Becker RC, Gebhard F, Mucher R, Scheible S, Nikolaus T. Epidemiologie von stürzen älterer. $\mathrm{Z} \mathrm{Or}$ thop 1999; 137(6):482-485.

48. Vellas BJ, Wayne SJ, Romero LJ, Baumgartner RN, Garry PJ. Fear of falling and restriction of mobility in elderly fallers. Age \& Aging 1997; 26(3):189-193.

49. Kong KS, Lee F, Mackenzie AE, Lee DTF. Psychosocial consequences of falling: the perspective of older Hong Kong chinese who had experienced recent falls. J Advanced Nursing 2002; 37(3):234-242.

50. Owings TM, Pavol MJ, Foley KT, Grabiner PC, Grabiner MD. Exercise: Is it a solution to falls by older adults? Journal of Applied Biomechanics 1999; 15(1):56-63.

51. Berg WP, Alessio HM, Mills EM, Tong C. Circunstances and consequences of falls in independent community - dwelling older adults. Age \& Ageing 1997; 26(4):261-268.

52. Rocha FL, Cunha UGV. Aspectos psicológicos e psiquiátricos das quedas do idoso. Arq Bras Med 1994; 68(1):9-13.

53. Campbell AG, Diep C, Reinken J, McCosh L. Factors predicting mortality in a total population sample of elderly. J Epidimiol Comm Health 1985; 39(4):337-342.

Artigo apresentado em 14/11/2011

Aprovado em 07/12/2011

Versão final apresentada em 08/12/2011 